\title{
Who wants to go further has to know the past
}

\section{A comment upon: Ultrasonography versus computed tomography for suspected nephrolithiasis-R. Smith-Bindman et al. N Engl J Med. 2014 Sep 18;371(12): 1100-1110}

\author{
François Luyckx
}

Received: 11 November 2014 / Accepted: 18 December 2014 / Published online: 28 December 2014

(C) Springer-Verlag Berlin Heidelberg 2014

The recent work by Smith-Bindman et al. [1] may influence future guidelines of emergency department practitioners, radiology department practitioners, and, maybe, urologists.

As this manuscript was published in a non-urological environment (i.e. N. Engl. J. M), the content might not draw the attention of urologist, as it was in my case at the first. But this manuscript has high impact on further management of abdominal pain in emergence department setting, although it completely lacks consensus with urological parties and their current guidelines and requirements.

Some issues with this work are listed below.

\section{Inclusion criteria}

The terms used in the text like "nephrolithiasis suspicion" are not valid. The manuscript talks about the differential diagnosis of "renal Colic" or "stone diathesis" with pain. The manuscript lacks typical terms and information about typical "urological" symptoms, such as referring pain to the outer genitalia or glans in Stone diathesis.

Only a half of the patients had costovertebral-angle tenderness. The other half was represented by lower quadrant tenderness. I therefore could assume that the diagnosis of «probable nephrolithiasis» may only have been influenced by the past history of nephrolithiasis $(40.4-43.1 \%$ of the patients in this study).

This kind of study, enrolling a large population, deserved an objective definition of the disease first. The use of scores, by example, would have been helpful.

\section{F. Luyckx $(\square)$}

Service d'Urologie, CHD Vendée, 85000 La Roche sur Yon, France

e-mail: poetschevleesch@hotmail.com
Recently, Moore et al. [2] built the STONE score, a 13-point scale including risk factors for uncomplicated ureteral stone. It encloses sex risk factors, origin, duration of pain to presentation, nausea, and haematuria.

This score, built upon a large series, confirms the relevance of clinical examination (patient history and physical examination) when evaluating the risk of nephrolithiasis in order to choose the proper imaging method.

Has the «likelihood of kidney stones» been calculated upon such a score or was it a subjective score written by the practitionists?

The «likelihood of kidney stones» was of $50 \%$ in 32.1 to $37.1 \%$ of the patients over the three groups.

It may be explained by the poor reliability of the symptoms used to include patients.

Furthermore, this may explain the unusual description of the population, far from the nephrolithiasis populations (as an example a M:F sex ratio of $1: 1$ in this study, $2: 1$ in the nephrolithiasis populations [2]).

\section{Reference standard}

$31.2-34.5 \%$ of the patients really had nephrolithiasis. It confirms the "likelihood of kidney stones". Otherwise, these quite low rates of true diagnosis emphasize the improper selection criteria of this study mentioned above.

Another issue is the definition of "nephrolithiasis" as a true diagnosis: it is written «either by the patient's observation of the passage of the stone or by the patient's report that the stone had been removed surgically». This definition excludes all the patients who missed the stone passage. It also includes those who believe they experienced a stone passage, but false (clot ....). The authors recognize this second limit. 


\section{Dosimetry}

One of the most important questions in this study was to limit radiation exposure in patients with suspected nephrolithiasis presenting in an emergency room.

The dosimetry is higher in the $« \mathrm{CT} »$ group at an average of $17.2 \mathrm{mSv}$ versus 10.1 and 9.3. $(p<0.001)$ in the both "US" groups.

Let us detail these data a bit.

We do not know how the average CT scanner units performed by each patient. We subsequently do not know the average dose given by each CT scanner.

It could only be deduced for the «CT» group. The average dose was $14.1 \mathrm{mSv}$, and this varied a lot $(\mathrm{SD}=9.6)$, probably because these CT scanners were performed according to «local standards». The signification of such a high per-CT dose may be explained by the non-use of lowdose CT scanners in this group and/or by the frequent use of contrast-enhanced CT with multiple acquisition protocols in a diagnostic intention because of diagnostic uncertainty. Indeed, if there is no stone in the non-contrast CT, other acquisitions are necessary to find the proper diagnosis (60\% or more patients in the CT group).

Nowadays, low-dose (7 mSv) and ultra-low-dose (1-2 mSv) CT scanners are more than promising diagnostic tools in renal colic. [3, 4].

Furthermore, in the ultrasonography groups, 27 and $40.7 \%$ of the patients needed additional diagnostic testing. Thus, the dosimetry remains lower in these groups, and we could imagine that the patients with hydronephrosis only needed non-contrast-enhanced CT scanner to ensure diagnosis of nephrolithiasis. How much of these CT scanners performed after an US were of low or ultra-low dose? This resulted in a lower radiation exposure and certainly blurred the interpretation of the data.

I should agree that the most appropriate method in order to avoid radiation consumption may relies in a strategy using ultrasonography first, followed by a low- or ultralow-dose non-enhanced CT scanner in patients with hydronephrosis on US.

\section{Why this commentary?}

It is mandatory for the urologist to know the mechanism of an acute renal colic, even uncomplicated. This point of view should not be forgotten when discussing the imaging protocol.

Let us imagine a patient with urothelial tumour risk factor(s) (cigarette smoking, professional exposure...). $\mathrm{He}$ is admitted in emergency room for acute renal colic. Ultrasonography finding ureterohydronephrosis, probable lower ureter obstacle. He leaves the emergency room with a symptomatic treatment of renal colic.

Let us imagine the same patient in my office one month later. No remaining pain, no experience of a stone passage, no evident hydronephrosis ... Should I consider he as cured and only order blood and urine tests to investigate lithiasis? No. Even if the risk of harbouring an upper urinary tract carcinoma is rare (incidence $=2 / 100000 / \mathrm{yr}$ ), this lifethreatening situation has not to be misdiagnosed.

My attitude is to perform contrast-enhanced CT scanner (4 acquisitions: no contrast-arterial-portal-urinary). A flexible cystoscopy would also be made to rule out any bladder tumour.

If he had a true 4-mm stone at the time of the renal colic, the dosimetry is clearly excessive. The only way to avoid such an over consumption of radiation is to identify the obstacle and its type with certitude at the time of the renal colic.

\section{Conclusion}

At this time, low- and ultra-low-dose CT scanners proved their reliability and may become the KUB of tomorrow, as an addition to the findings of ultrasonography, and not versus ultrasonography alone.

The point of view of an urologist certainly would have allowed the authors to consider the renal colic as the beginning of a long-standing disease: nephrolithiasis. It is now well established that nephrolithiasis deserves the most appropriate initial description (stone size, number, location, spectroscopic analysis, 24-h urine and blood tests...) to allow the urologist to proper inform patients for prophylaxy of recurrences.

\section{References}

1. Smith-Bindman R, Aubin C, Bailitz J, Bengiamin RN, Camargo CA, Corbo J et al (2014) Ultrasonography versus computed tomography for suspected nephrolithiasis. N Engl J Med 371(12):1100-1110

2. Moore CL, Bomann S, Daniels B, Luty S, Molinaro A, Singh D et al (2014) Derivation and validation of a clinical prediction rule for uncomplicated ureteral stone-the STONE score: retrospective and prospective observational cohort studies. BMJ 348:g2191

3. McLaughlin PD, Murphy KP, Hayes SA, Carey K, Sammon J, Crush L et al (2014) Non-contrast CT at comparable dose to an abdominal radiograph in patients with acute renal colic; impact of iterative reconstruction on image quality and diagnostic performance. Insights Imaging 5(2):217-230

4. Drake T, Jain N, Bryant T, Wilson I, Somani BK (2014) Should low-dose computed tomography kidneys, ureter and bladder be the new investigation of choice in suspected renal colic?: A systematic review. Indian J Urol Soc 30(2):137-143 